\title{
Right-wing authoritarianism, fundamentalism and prejudice revisited: Removing suppression and statistical artefact
}

\author{
Kenneth I. Mavor ${ }^{\mathrm{a}, *}$, Cari J. Macleod ${ }^{\mathrm{a}}$, Miranda J. Boal ${ }^{\mathrm{a}}$, Winnifred R. Louis ${ }^{\mathrm{b}}$ \\ a School of Psychology, The Australian National University, Canberra, ACT 0200, Australia \\ ${ }^{\mathrm{b}}$ School of Psychology, University of Queensland, St. Lucia, QLD 4072, Australia
}

\section{A R T I C L E I N F O}

\section{Article history:}

Received 24 May 2008

Received in revised form 18 October 2008

Accepted 18 December 2008

Available online 29 January 2009

\section{Keywords:}

Right-wing authoritarianism

Fundamentalism

Prejudice

Religiosity

Statistical artefact

\begin{abstract}
A B S T R A C T
This paper challenges a finding reported by several researchers, that fundamentalism could be associated with a reduction in racial prejudice after controlling for authoritarianism (RWA). We argue that the presence of fundamentalism in the construct definition of the conventionalism cluster of RWA leads to higher associations between fundamentalism and conventionalism than with other aspects of RWA. This creates a statistical artefact that distorts the results of multiple regression analyses that include both fundamentalism and RWA as independent variables. To test this hypothesis, 299 participants completed measures of prejudice as well as fundamentalism and the three RWA clusters (conventionalism, authoritarian aggression, and submission). In regression analyses using fundamentalism and the combined RWA scale we replicate previous findings that when RWA is controlled, higher fundamentalism leads to lower prejudice. After removing the overlapping method variance in the scales, this pattern is eliminated and the commonly observed positive relationship between fundamentalism and prejudice is found. We describe the statistical artefact, its antecedents, and its theoretical implications, and outline how investigations in this important area should proceed.
\end{abstract}

(c) 2009 Elsevier Ltd. All rights reserved.

\section{Introduction}

Would fundamentalism actually lead to reduced prejudice if the authoritarian component could be controlled? This is the important implication of several studies which consider the impact of an individual's Christian fundamentalism and right-wing authoritarianism (RWA; Altemeyer, 1981, 1988, 1996) on racial prejudice and homosexual prejudice (e.g., Laythe, Finkel, Bringle, \& Kirkpatrick, 2002; Laythe, Finkel, \& Kirkpatrick, 2001; Rowatt \& Franklin, 2004). Both fundamentalism and RWA typically have positive zeroorder correlations with prejudice. However, when entered jointly in multiple regression, fundamentalism may have a negative beta weight for racial prejudice and a less positive or non-significant link to homosexual prejudice. This effect is of great potential interest to researchers in the social psychology of religion and prejudice (see e.g., Hunsberger \& Jackson, 2005). However, we argue that before this can be interpreted in a substantive way we must consider the alternative possibility that it represents a statistical artefact arising from the complex nature of the RWA construct and partial overlap between RWA and fundamentalism.

Individual differences in authoritarianism and religiosity have long been associated with prejudiced attitudes. Across a range of social contexts, the Right-wing Authoritarianism scale correlates

\footnotetext{
* Corresponding author. Tel.: +61261250639 (w).

E-mail address: Ken.Mavor@anu.edu.au (K.I. Mavor).
}

with various forms of prejudice (e.g., Altemeyer, 1996; Crowson, DeBacker, \& Thoma, 2005; Heaven, Organ, Supavadeeprasit, \& Leeson, 2006; Heaven \& St. Quintin, 2003; Mirisola, Sibley, Boca, \& Duckitt, 2007). In the study of religion and prejudice, fundamentalism has emerged as a central construct (e.g., Altemeyer \& Hunsberger, 1992; Hunsberger, 1995), and, together with RWA, large amounts of variance can be consistently explained (see also, Laythe et al., 2001, 2002; Wylie \& Forest, 1992). However, combining RWA, fundamentalism and certain targets of prejudice is problematic, we argue, and can lead to potentially incorrect interpretations in correlational multivariate analyses, such as multiple regression.

\subsection{RWA and overlapping constructs}

The main source of the problem is the complex construct definition and measurement of RWA. RWA is an individual difference construct that represents the covariation of three underlying clusters: authoritarian aggression, authoritarian submission, and conventionalism (Altemeyer, 1981, 1988, 1996). Although researchers recognise the importance of distinguishing the underlying clusters (e.g., Funke, 2005; Manganelli Rattazzi, Bobbio, \& Canova, 2007; Smith \& Winter, 2002), the design of the RWA scale makes it difficult for the three components to emerge separately. Items were designed by Altemeyer to tap into multiple clusters emphasising the central construct and diluting the differences between clusters. Wording direction also conceals the factor 
structure, since aggression items tend to be worded in a construct positive direction, and conventionalism items in a construct negative direction (Duckitt \& Fisher, 2003; Funke, 2005; Manganelli Rattazzi et al., 2007). Accordingly, a two-factor solution is sometimes discarded as representing method factors (e.g., Altemeyer, 1996).

In the present paper, we describe two fundamental statistical problems that may arise when researchers ignore the complex structure of RWA and its partial overlap with other constructs. The first problem is the inflation of apparent relationships due to correlating part-whole measures. The conventionalism cluster within RWA contains items measuring homosexual prejudice, traditional roles of women, fundamentalist approaches to religion, and conservative attitudes to sexuality. When RWA is correlated with other scales measuring these other attitudes as targets, the true construct relationships are inflated by the measurement variance to an unknown extent (Whitley \& Lee, 2000).

The second form of statistical problem occurs in the more complex case where the full RWA scale is used in a regression analysis alongside another independent variable (e.g., fundamentalism) that is measured within the RWA scale, and therefore also subject to the part-whole problem raised above. However in this case, part of the correlation between RWA and the other independent variable is due to overlapping method variance. This may lead to incorrect estimation of the beta weights in the model and subsequent incorrect conclusions about the relationships observed in empirical data, as elaborated below.

\subsection{The emergence of the artefact in the social psychological literature}

The problems caused by overlap between RWA and other predictors of prejudice remain hidden when analyses are primarily based on zero-order correlations. Since strong correlations are expected between RWA, fundamentalism, homosexual prejudice, and racial prejudice, there has been no reason to challenge their association in several previous studies (e.g., Altemeyer \& Hunsberger, 1992; Wylie \& Forest, 1992). It was only when Laythe et al. (2001) focused our attention on a predictive model in which RWA and fundamentalism were jointly used to predict homosexual prejudice and racial prejudice that the particular pattern of interest emerged.

Laythe et al. (2001) present an analysis on their own dataset, and re-analyse the data from two previous studies: Altemeyer and Hunsberger (1992), and Wylie and Forest (1992). In all three cases they regress two dependent variables, homosexual prejudice and racial prejudice, on RWA and fundamentalism. The results for all three analyses are presented in parallel in Fig. 1. For racial prejudice, fundamentalism shows a negative path coefficient, suggesting that fundamentalism reduces racial prejudice after controlling for RWA. For homosexual prejudice, RWA is clearly the stronger predictor, and although the path from fundamentalism remains positive, it is not significantly different from zero in two of the three analyses.

This pattern of results led Laythe and colleagues to conclude that associations previously found between fundamentalism and prejudice were largely due to authoritarianism, and that, after controlling for RWA, the association between fundamentalism and prejudice was reduced or even reversed. Specifically, Laythe et al., argued that:

In none of the three data sets was the fundamentalism-racial prejudice relationship significantly positive when RWA was controlled. However, our results for racial prejudice went beyond this: in all three data sets, fundamentalism not only failed to be a positive predictor, but it was a significant inverse predictor of racial prejudice. The consistency of results across
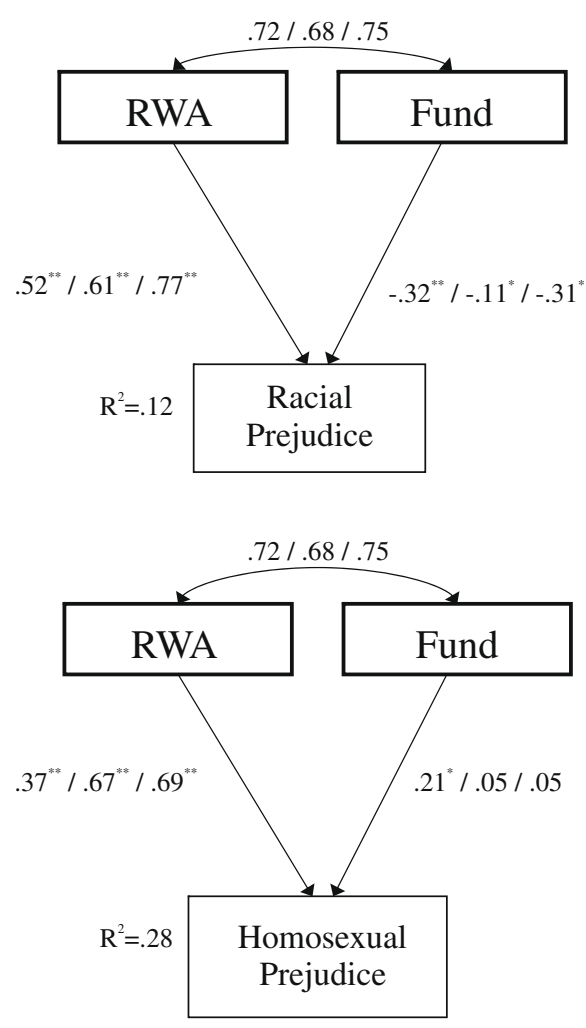

$$
* \mathrm{p}<.05, * * \mathrm{p}<.01, * * * \mathrm{p}<.001
$$

Fig. 1. Path weights for the three data sets analysed in Laythe et al. (2001). The first set of path weights are those reported for their own data (study 1). The second and third paths weights in each position are from Altemeyer and Hunsberger (1992), and Wylie and Forest (1992), respectively. The $\mathrm{R}^{2}$ figures come from the Laythe et al. study 1 .

the three studies - our own data (Study 1) and the reanalysis of two previously published data sets (Study 2) - suggests that this is a real effect that must be explained. (Laythe et al., 2001, p. 7)

\section{A critique.}

We contend that both patterns of regression weights could be the consequence of the inflated overlap between the RWA and fundamentalism variables in the regression. In particular, the suppression effect for fundamentalism and racial prejudice may illustrate this problem most clearly. A negative suppression effect refers to a change in the direction (positive or negative) of the relationship between an independent variable and a dependent variable when a third factor (the suppressor) is controlled. The effect of fundamentalism on prejudice meets the criterion for negative suppression because the zero-order correlation between fundamentalism and prejudice is positive, but the beta coefficient of fundamentalism on prejudice is negative when RWA is controlled. Maassen and Bakker (2001) describe the conditions when negative suppression will arise as follows:

Although the suppressor has relevant information in common with Y, they share fewer common elements than the common elements of irrelevant information shared by the suppressor and the other predictor. (Maassen \& Bakker, 2001, p. 245)

To restate this formal definition into terms of our focal variables: although fundamentalism has relevant information in common with racial prejudice, this is smaller than the shared 
information between RWA and fundamentalism that is irrelevant to the prediction of racial prejudice. We argue that the shared information between RWA and fundamentalism that is irrelevant to the prediction of racial prejudice is due to the overlap between fundamentalism and the conventionalism component of RWA. If this is the case, then the negative suppression effect is a statistical artefact and not a substantive finding needing explanation.

\subsection{The present study}

To address this question empirically, we can measure the separate components of RWA to remove overlapping measurement with fundamentalism and homosexual prejudice. Several researchers have created shortened subscale measures from the full RWA scale based on face validity grounds (Duncan, Peterson, \& Winter, 1997; Funke, 2005; Manganelli Rattazzi et al., 2007; Smith \& Winter, 2002). These researchers have found that the shorter scales capturing the three theorised components of RWA do a good job of capturing the overall RWA construct. In the present study, we adopted the same approach, using items identified by Smith and Winter (2002) as indicators of the three components. Their three component measures correlated from .70 to .82 with the full 30 item RWA scale. These correlations underestimate the true strength of the relationship between the components and the full scale because of measurement error and the lower reliability of short scales. After correcting for the attenuation of correlation due to measurement error (Nunnally, 1978), the estimated construct correlations ranged from .92 to 1.0 .

Our first goal is to test the hypothesis that the construct overlap between conventionalism and fundamentalism is a key explanatory factor of past findings. We evaluate this in several ways: examining the zero-order correlations; computing the estimated construct correlations between all measures (Nunnally, 1978); and carrying out an item-analysis of the RWA and fundamentalism measures. The construct correlations are estimates of the correlations to be expected if all the variables were measured without error (i.e., fully correcting for the estimated attenuation of correlation due to imperfect scale reliability). The overlap between RWA and fundamentalism will be seen to be problematic if raw and adjusted correlations between fundamentalism and RWA conventionalism are as high, or higher, than those between conventionalism and other components of RWA, and if item-analysis suggests that conventionalism items load more highly with fundamentalism items than other RWA items.

Subsequently, we will conduct multiple regressions in which homosexual and racial prejudice are predicted by fundamentalism and the combined RWA scale (replicating past problematic research) versus by fundamentalism and authoritarian aggression, eliminating the conventionalism cluster. Authoritarian aggression is the component with the strongest link with prejudice in past research (Altemeyer, 1996; Manganelli Rattazzi et al., 2007). If authoritarian aggression is the key component of the RWA scale that predicts prejudice, controlling for RWA aggression instead of the combined scale would result in negligible differences in the variance accounted for.

Moreover, if the negative beta coefficient for fundamentalism on racial prejudice controlling for RWA is an artefact due to the overlap of fundamentalism and the conventionalism component of RWA, then controlling for RWA aggression instead of the full scale will eliminate the suppression effect, resulting in a positive beta for fundamentalism more consistent with the positive zeroorder correlation of fundamentalism and racial prejudice. Conversely, if Laythe et al. (2001) are correct that fundamentalism would have the effect of reducing racial prejudice if the effects of authoritarianism on prejudice are removed, then controlling for the most potent component of RWA should leave the negative fundamentalism-prejudice relationship the same or even strengthened.

The predictions for homosexual prejudice are more complex because of the presence of homosexuality items, as well as fundamentalism items, in the conventionalism cluster. Since the RWA aggression items do not directly tap homosexual prejudice, whereas the combined scale does, we would expect the overall variance accounted for to be reduced when the subscale is substituted for the combined scale, and the size of the beta coefficient for RWA to decrease. If the main reason that the combined RWA scale seems to be the stronger predictor of homosexual prejudice is the presence of the both homosexual prejudice and fundamentalism items in the scale, then we would also expect that when the combined RWA scale is replaced by the aggression component only, the fundamentalism scale would show an increased path weight, and RWA would show a reduced path weight.

\section{Method}

\subsection{Participants}

A total of 299 participants were recruited for the study. To obtain a reasonable sample size and to ensure good variability on all the measures we used a mixture of university and community samples. Ninety-four active Christian participants were recruited from a variety of local church congregations through visits by the researchers or through contacts with the clergy, and a further 205 participants were members of a first-year political science class who volunteered to participate at the end of a lecture class. Of the political science students, 78 reported identifying as Christian, giving 172 Christian-identified participants overall. The sample included 107 men and 191 women (with 1 unknown) and a mean (S.D.) age of 23.2 (11.2).

\subsection{Procedure}

The "Attitudes to Social Issues Questionnaire" was administered to the students during a lecture and collected immediately. In the case of the church sample, some questionnaires were completed at the time they were distributed, while others were collected a week later or were returned by mail. All questionnaires were completed anonymously and took approximately $15 \mathrm{~min}$ to complete.

\subsection{Materials}

The scales utilised in this study were part of a larger questionnaire ${ }^{1}$. The RWA scale consisted of 10 items. Nine of the items were those used by Smith and Winter (2002), of which three measured authoritarian aggression (e.g., "What our country really needs is a strong determined leader who will crush evil, and take us back to our true path"), three measured authoritarian submission (e.g., "What our country needs most is discipline, with everyone following our leader in unity."), and three measured conventionalism, the most contentious subscale ("Gays and lesbians are just as healthy and moral as anybody else."; "Everyone should have their own lifestyle, religious beliefs, and sexual preferences, even if it makes them different from everyone else."; People should pay less attention to the Bible and other old traditional forms of religious guidance, and instead develop their own personal standards of what is moral and immoral."). Since the first conventionalism item is explicitly about homosexual prejudice, we wanted to see the effect of excluding this single item on prediction. To be able to maintain at least three items in each scale we added a fourth conventionalism item, taken from

\footnotetext{
${ }^{1}$ Available from the lead author upon request.
} 
Table 1

Reliability, means, standard deviations and correlations for RWA, fundamentalism and attitude variables.

\begin{tabular}{|c|c|c|c|c|c|c|c|c|c|}
\hline & Mean (SD) & Alpha & 1 & 2 & 3 & 4 & 5 & 6 & 7 \\
\hline 1. Fundamentalism & $2.99(1.55)$ & .93 & - & $.82^{* * *}$ & $.49^{* * *}$ & $.62^{* * *}$ & $.87^{* * *}$ & $.79^{* * *}$ & $.35^{* * *}$ \\
\hline 2. RWA & $2.77(1.19)$ & .87 & .91 & - & $.79^{* * *}$ & $.86^{* * *}$ & $.89^{* * *}$ & $.82^{* * * *}$ & $.50^{* * *}$ \\
\hline 3. RWA Aggression & $2.69(1.27)$ & .69 & .61 & 1.0 & - & $.67^{* * *}$ & $.49^{* * *}$ & $.55^{* * *}$ & $.48^{* * *}$ \\
\hline 4. RWA Submission & $2.52(1.18)$ & .65 & .80 & 1.0 & 1.0 & - & $.63^{* * *}$ & $.62^{* * * *}$ & $.45^{* * *}$ \\
\hline 5. RWA Conventionalism & $3.03(1.65)$ & .83 & .99 & 1.0 & .64 & .85 & - & $.83^{* * *}$ & $.38^{* * *}$ \\
\hline 6. Homosexual Prejudice & $5.40(1.54)$ & .80 & .91 & .98 & .74 & .86 & 1.0 & - & $.47^{* * *}$ \\
\hline 7. Racial Prejudice & $5.51(1.15)$ & .83 & .39 & .59 & .64 & .62 & .46 & .58 & - \\
\hline
\end{tabular}

$p<.001$; Upper diagonal presents raw correlations, lower diagonal presents construct correlations (corrected for attenuation).

the full RWA scale: "There is nothing wrong with premarital sexual intercourse". All the conventionalism items are reverse-scored (as in the full RWA scale).

Fundamentalism was measured using a 10-item scale with five items drawn from McFarland's (1989) version of the scale (e.g., "It is very important for true Christians to believe that the bible is the infallible word of God."), and five from Altemeyer and Hunsberger (1992; e.g., "Whenever science and sacred scripture conflict, science must be wrong."). The two criterion scales were racial attitudes (six items measuring attitudes to Aboriginal Australians and immigrants adapted from Modern Racism; e.g., "Aboriginal Australians are getting too demanding in their push for rights."; McConahay, Hardee, \& Batts, 1981) and attitudes to gay men and lesbians (four items; e.g., "If a man has homosexual feelings, he should do everything he can to overcome them."; Herek, 1984). Participants responded to all items on a 7-point Likert scale ranging from strongly disagree to strongly agree with a neutral mid-point.

\section{Results and discussion}

\subsection{Preliminary analyses}

The means, standard deviations and scale reliabilities for all measures are shown in Table 1 . There were no problems with skewness, non-linearity or heteroscedasticity.

\subsection{Correlations}

The correlations between all variables in the study are shown in the upper triangle of Table 1. Consistent with previous findings, RWA and fundamentalism are highly correlated. Fundamentalism is most highly correlated with the conventionalism subscale and least correlated with the aggression subscale. Both scales have moderate relationships with prejudice. Higher scores on both fundamentalism and RWA (and all subscales) are associated with increased prejudice.

\subsection{Construct correlations}

Since we are comparing correlations among scales of varying length and reliability we also present construct correlations (Nunnally, 1978) in the lower triangle of Table 1 , which represent the relationship between the scales after adjusting for different reliabilities. Conventionalism, fundamentalism, and homosexual prejudice are correlated as strongly as their measurement reliability allows. In short, there is more variance in common between the conventionalism scale and the associated constructs of fundamentalism and homosexual prejudice, than there is between conventionalism and the other components of RWA.

\subsection{Item analysis}

An exploratory common factor analysis was conducted on the ten RWA items and the ten fundamentalism items, using an obli- min rotation. Two common factors were extracted, based on eigenvalues greater than one and the scree-plot criterion. The first factor was defined primarily by the fundamentalism items and the four conventionalism items which had loadings on the first factor ranging from .51 to .89 . The second factor was defined by the three RWA aggression items, and one of the RWA submission items. The remaining two RWA submission items had crossed loadings on both factors. The factors themselves correlated .62 .

This analysis supports the view that the RWA conventionalism items are more strongly related to the fundamentalism items than they are to the other parts of the RWA scale. At the item-level the data support the patterns evident from scale correlations and estimated construct correlations, i.e., that the overlap between fundamentalism and RWA conventionalism undermines both the notion of RWA as a unitary construct in which the three components covary equally, and the notion that fundamentalism and RWA are operationalized as distinct independent variables.

\subsection{Multiple regression analyses}

To replicate the results found by Laythe and colleagues (2001), we carried out a series of multiple regression analyses with fundamentalism and RWA as predictors on each criterion variable. The results of these analyses can be seen in the top panels of Figs. 2 and 3. Both RWA and fundamentalism have positive beta weights when predicting homosexual prejudice with the larger beta coefficient belonging to RWA (.52 compared to .36). When considering racial prejudice, the beta weight for RWA is positive (.65) but the beta for fundamentalism is negative $(-.19)$. This is the same pattern obtained by Laythe and colleagues that led them to conclude that, after controlling for RWA, higher scores on fundamentalism are associated with more positive views toward racial groups.

We now consider a parallel set of analyses, substituting the RWA aggression subscale in the analysis in place of the combined RWA scale (see bottom panels of Figs. 2 and 3). The RWA aggression scale had an estimated construct correlation of 1.0 with the overall RWA scale, indicating that the aggression scale is as good a representation of the overall construct as possible given their reliabilities. As predicted, overall $R^{2}$ values predicting prejudice are nearly identical when the aggression component is employed compared to the combined RWA scale: $66 \%$ vs. $71 \%$ of the variability in homosexual prejudice and $25 \%$ vs. $26 \%$ of the variability in racial prejudice.

The drop in prediction is particularly interesting to unpack in the case of homosexual prejudice, since the 10-item RWA scale includes an item that is an explicit measure of homosexual prejudice. In addition we expect some drop in prediction for both criterion variables due to the lower reliability of the three-item aggression measure. To explore this further we conducted a regression analysis to predict homosexual prejudice using fundamentalism plus a nine-item RWA scale with the explicit homosexual prejudice item removed. This led to a drop in prediction from $71 \%$ to $69 \%$. Thus changing the measurement of RWA to exclude the overlapping item content between homosexual prejudice and RWA only effec- 


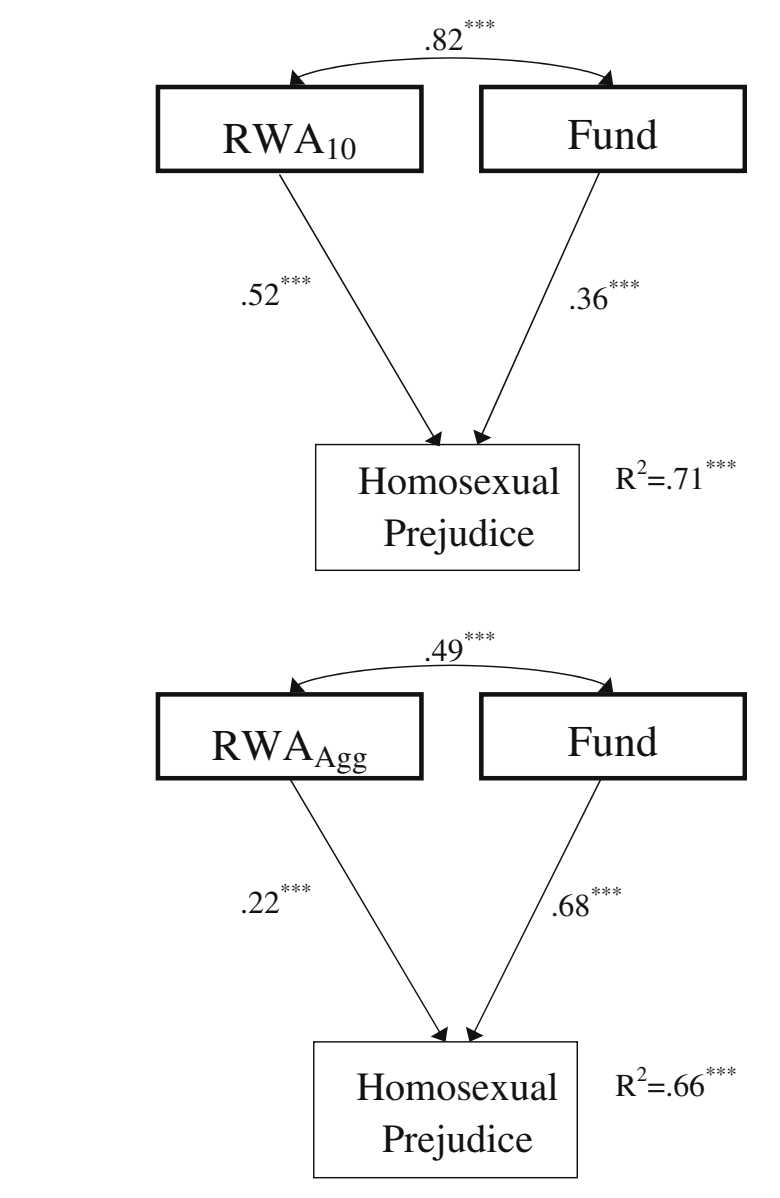

$* \mathrm{p}<.05, * * \mathrm{p}<.01, * * * \mathrm{p}<.001$

Fig. 2. Regression weights when predicting homosexual prejudice based on fundamentalism and RWA. RWA in the top panel is represented by all three subcomponents of RWA as measured via our 10-item scale. RWA in the bottom panel is represented by the RWA aggression subscale alone.

tively drops the prediction by $2 \%$. We then used the formula from Nunnally (1978) for estimating the reliability of a linear composite to estimate the further expected drop in prediction due to reduced reliability with the 3 - versus 9 -item scale. This formula dropped the expected prediction to $68 \%$, compared to the final regression prediction of $66 \%$.

Overall very little power has been lost in the ability to predict either racial or homosexual prejudice, yet the correlations between fundamentalism and RWA have dropped from .82 (fundamentalism with combined RWA) to .49 (fundamentalism with RWA aggression). That is, the overlap between the predictors dropped from $67 \%$ to $24 \%$, a reduction in overlapping variance between IVs of $43 \%$, while the effective drop in the variance accounted for in the dependent variables is approximately $1-2 \%$ for both racial and homosexual prejudice. These findings support the conclusion that the overlap between fundamentalism and the combined RWA scale is largely unrelated to the criterion variables.

When we turn to the beta weights, we see the conclusive evidence that the suppression effect for fundamentalism is an artefact of its correlation with conventionalism. When RWA aggression is controlled, fundamentalism is revealed as a strong predictor of homosexual prejudice (.68, compared with .22 for RWA aggression). Fundamentalism is also a positive predictor of racial prejudice (.15, compared to .41 for aggression), eliminating the negative suppression effect found in analyses with the combined RWA scale. Consistent with its zero-order correlations, higher fun-
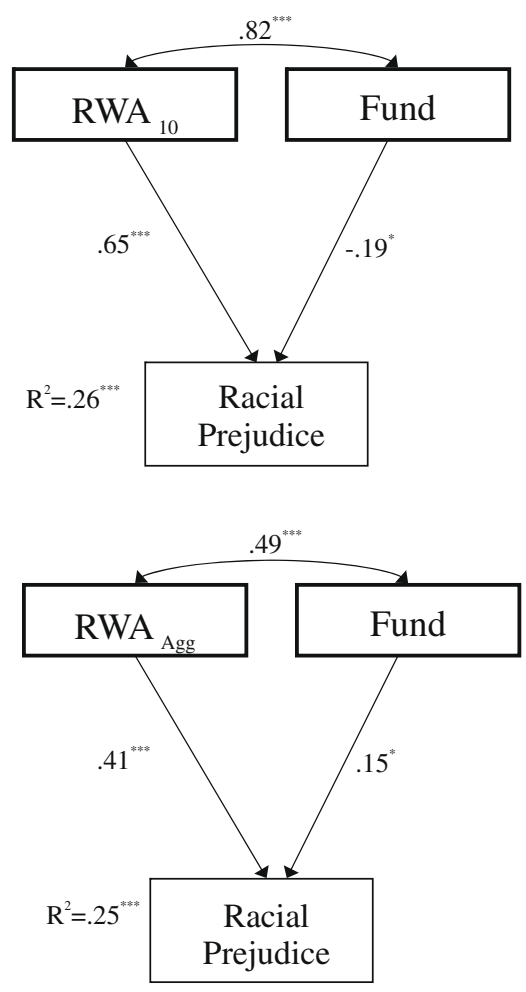

${ }^{*} \mathrm{p}<.05, * * \mathrm{p}<.01, * * * \mathrm{p}<.001$

Fig. 3. Regression weights when predicting racial prejudice based on fundamentalism and RWA. RWA in the top panel is represented by all three subcomponents of RWA as measured via our 10-item scale. RWA in the bottom panel is represented by the RWA aggression subscale alone.

damentalism is associated with more homosexual and racial prejudice, even when authoritarian aggression is controlled.

The changes in beta weights take a different form for the two criterion variables. For racial prejudice, the effect of fundamentalism changes from a negative beta (in a model with full RWA) to a positive beta, eliminating the suppression effect (in a model with RWA aggression); however, RWA remains the stronger predictor. For homosexual prejudice the beta weights remain positive for fundamentalism, but the priority changes such that RWA appears to be the stronger predictor in the analysis with the composite scale, but fundamentalism becomes the stronger predictor once the construct overlap is removed. Both of these changes represent a common underlying process. In both cases, RWA starts with a higher zero-order correlation with the criterion than fundamentalism and will therefore have a higher beta weight. The inflated correlation between RWA and fundamentalism caused by the overlap of fundamentalism with conventionalism amplifies this higher beta, forcing the other beta weight to be reduced to compensate. A beta weight represents the change in the dependent variable associated with one standard deviation shift in an independent variable, holding constant all other independent variables. Holding constant RWA when examining the impact of fundamentalism on prejudice requires that the increase in conventionalism which is associated with higher fundamentalism be offset within RWA with compensating drops in the other components, such as aggression. Since aggression is correlated with prejudice, the negative beta for fundamentalism, while holding constant RWA, reflects the artefactual drop in the aggression component, which is associated with lower prejudice scores. In the case of racial prejudice the adjustment is enough to drive the beta for fundamentalism into the negative range. In the case of homosexual prejudice, the same removal 
of the conventionalism component (which included both homosexual prejudice and fundamentalism within it) changes the pattern of zero-order correlations such that fundamentalism now shows the stronger relationship with prejudice compared to RWA aggression (see Table 1). This is consistent with the change in priority of prediction in the regression, such that fundamentalism becomes the strongest predictor when RWA aggression is controlled.

\section{Conclusion}

The analyses we have presented here lead to a very clear conclusion. It is possible to have fundamentalism appear to reduce prejudice after controlling for authoritarianism using the combined RWA scale. However, the effect is very likely due to overlapping variance between the RWA and fundamentalism measures. To show that it was the association of conventionalism and fundamentalism that was causing the potentially misleading findings, we established several key results: (1) construct correlations and item-level factor analysis demonstrated that the connections between fundamentalism and RWA conventionalism were stronger than the associations between conventionalism and aggression aspects of RWA. (2) In regression analyses with fundamentalism and the combined RWA construct, fundamentalism appeared to lead to reduced prejudice, in a pattern replicating earlier research. (3) When the same regressions involved fundamentalism and RWA aggression only, the overlap between the predictor constructs fell by $43 \%$ but the proportion of the dependent variables predicted effectively fell by only $1-2 \%$. (4) Once the overlapping variance was removed, fundamentalism was associated with increased racial prejudice and homosexual prejudice in a pattern consistent with the positive zero-order correlations.

It is still possible that fundamentalism could be associated with reduced prejudice after controlling for RWA or other factors, but our analyses cast doubt on the existing findings by researchers who have included the full RWA scale and fundamentalism in regression analyses (e.g., Laythe et al., 2002; Rowatt \& Franklin, 2004; Wylie \& Forest, 1992). We believe that to explore the relationship between fundamentalism and authoritarianism correctly requires an analysis that is capable of distinguishing the underlying clusters within the RWA construct. This is difficult with current measures of RWA, but several approaches are possible, either using face-valid subsets of Altemeyer's RWA items (e.g., Smith \& Winter, 2002) or using newly developed measures of the separate clusters (Funke, 2005). Once these issues are resolved, then researchers can turn their attention with more confidence to exploring the important role of fundamentalism and authoritarianism in understanding the nature of prejudice.

\section{References}

Altemeyer, B. (1981). Right-wing authoritarianism. Winnipeg, Canada: University of Manitoba Press.

Altemeyer, B. (1988). Enemies of freedom: Understanding right-wing authoritarianism. San Francisco: Jossey-Bass.

Altemeyer, B. (1996). The authoritarian specter. Cambridge, Massachusetts: Harvard University Press.

Altemeyer, B., \& Hunsberger, B. E. (1992). Authoritarianism, religious fundamentalism, quest, and prejudice. International Journal for the Psychology of Religion, 2, 113-133.

Crowson, H., DeBacker, T. K., \& Thoma, S. J. (2005). Does authoritarianism predict post-9/11 attitudes? Personality and Individual Differences, 39, 1273-1283.

Duckitt, J., \& Fisher, K. (2003). The impact of social threat on worldview and ideological attitudes. Political Psychology, 24, 199-222.

Duncan, L. E., Peterson, B. E., \& Winter, D. G. (1997). Authoritarianism and gender roles: Toward a psychological analysis of hegemonic relationships. Personality $\mathcal{E}$ Social Psychology Bulletin, 23, 41-49.

Funke, F. (2005). The dimensionality of right-wing authoritarianism: Lessons from the dilemma between theory and measurement. Political Psychology, 26, 195-218.

Heaven, P. C., Organ, L.-A., Supavadeeprasit, S., \& Leeson, P. (2006). War and prejudice. A study of social values, right-wing authoritarianism, and social dominance orientation. Personality and Individual Differences, 40, 599-608.

Heaven, P. C., \& St. Quintin, D. (2003). Personality factors predict racial prejudice. Personality and Individual Differences, 34, 625-634.

Herek, G. M. (1984). Attitudes toward lesbians and gay men: A factor analytic study. Special Issue: Homophobia: An overview. Journal of Homosexuality, 10, 39-51.

Hunsberger, B. (1995). Religion and prejudice. The role of religious fundamentalism, quest, and right-wing authoritarianism. Journal of Social Issues, 51, 113-129.

Hunsberger, B., \& Jackson, L. M. (2005). Religion, meaning, and prejudice. Journal of Social Issues, 61, 807-826.

Laythe, B., Finkel, D. G., Bringle, R. G., \& Kirkpatrick, L. A. (2002). Religious fundamentalism as a predictor of prejudice. A two-component model. Journal for the Scientific Study of Religion, 41, 623-635.

Laythe, B., Finkel, D. G., \& Kirkpatrick, L. A. (2001). Predicting prejudice from religious fundamentalism and right-wing authoritarianism: A multiple regression approach. Journal for the Scientific Study of Religion, 40, 1-10.

Maassen, G. H., \& Bakker, A. B. (2001). Suppressor variables in path models: Definitions and interpretations. Sociological Methods E Research, 30, 241-270.

Manganelli Rattazzi, A. M., Bobbio, A., \& Canova, L. (2007). A short version of the right-wing authoritarianism (RWA) scale. Personality and Individual Differences, 43, 1223-1234.

McConahay, J. B., Hardee, B. B., \& Batts, V. (1981). Has racism declined in America? Journal of Conflict Resolution, 25, 563-579.

McFarland, S. G. (1989). Religious orientations and the targets of discrimination. Journal for the Scientific Study of Religion, 28, 324-336.

Mirisola, A., Sibley, C. G., Boca, S., \& Duckitt, J. (2007). On the ideological consistency between right-wing authoritarianism and social dominance orientation. Personality and Individual Differences, 43, 1851-1862.

Nunnally, J. C. (1978). Psychometric theory (2nd ed.). New York: McGraw Hill.

Rowatt, W. C., \& Franklin, L. M. (2004). Christian orthodoxy, religious fundamentalism, and right-wing authoritarianism as predictors of implicit racial prejudice. International Journal for the Psychology of Religion, 14, 125-138.

Smith, A. G., \& Winter, D. G. (2002). Right-wing authoritarianism, party identification, and attitudes toward feminism in student evaluations of the Clinton-Lewinsky story. Political Psychology, 23, 355-383.

Whitley, B. E., Jr., \& Lee, S. E. (2000). The relationship of authoritarianism and related constructs to attitudes toward homosexuality. Journal of Applied Social Psychology, 30, 144-170.

Wylie, L., \& Forest, J. (1992). Religious fundamentalism, right-wing authoritarianism and prejudice. Psychological Reports, 71, 1291-1298. 